\section{Deep grey matter involvement in multiple sclerosis: key player or bystander?}

\author{
Izumi Kawachi
}

Multiple sclerosis (MS) has been classically considered to be an autoimmune and inflammatory demyelinating disease that primarily affects the white matter (WM) in the central nervous system. However, because WM demyelination by itself cannot explain the full extent of clinical disabilities including cognitive impairments in patients with MS, renewed interest in grey matter (GM) pathology is now emerging. ${ }^{1-4}$

GM lesions in MS are found in both cortical GM and deep GM by pathological and radiological studies. A 3D double inversion recovery technique on MRI enables a fivefold increase in the detection of cortical GM lesions, which are regarded as a better predictor of cognitive impairment than WM lesions in patients with MS. The cortical GM pathology is characterised as follows: (i) band-like subpial demyelination, invariably oriented towards the pial surface of the cortex with variable depths, is the most common type; (ii) it is particularly widespread in deep infoldings of the brain surface in association with inflammatory infiltrates in the meninges; (iii) it is mainly found in patients with progressive MS, but is also present in patients with early-stage $\mathrm{MS}^{5}$ (iv) typical pathological hallmarks of WM lesions, including lymphocyte and foamy macrophage infiltrations, complement deposition, microglial activation and blood-brain barrier disruption, are unusual; and (v) cortical GM is affected by two processes, demyelination and neuroaxonal degeneration.

In contrast to progress in cortical GM research in MS, previous studies analysing deep GM by using radiological or pathological methods have been limited by their

Correspondence to Dr Izumi Kawachi, Department of Neurology, Brain Research Institute, Niigata University, 1-757 Asahimachi, Chuo-ku, Niigata 951-8585, Japan; ikawachi@bri.niigata-u.ac.jp small sample size, registration biases, retrospective design and the absence of treatment effects on deep GM lesions over the course, and details of deep GM pathology remain unclear. Haider et $a l^{6}$ provide us with pathological evidence of deep GM involvement in MS with a focus on the incidence and topographical distribution of lesions in relation to WM and cortical GM on a total sample of $75 \mathrm{MS}$ archival autopsy patients and 12 controls. Haider et $a l^{6}$ highlight the significance of the caudate nucleus and hypothalamus as deep GM demyelinating lesions, an intermediate inflammation phenotype between low inflammatory cortical GM lesions and active WM lesions, and pathogenic processes of which include demyelination, neurodegeneration and accumulation of oxidative injury in deep GM pathology. Their data can contribute to our understanding of the topography and pathogenic mechanisms of deep GM involvement in MS. However, further investigation is necessary to resolve the following problems. First, since the study was performed on archival autopsied materials and the sampling of deep GM blocks in the tissue was not systemically standardised enough to analyse the details of region-dependent deep GM pathology (eg, dominant location within different deep GM nuclei or subnuclei), most of their quantitative analyses lumped different deep GM nuclei or subnuclei into the same category as 'deep GM'. Further topographic evaluation focusing on each deep GM nucleus or subnucleus would be important for discovery of new pathogenic mechanisms. Second, the cause of damage in deep GM, which consists of focal demyelination and global neuroaxonal degeneration, still remains unknown. Third, there has been no clear and direct evidence that global neurodegeneration in deep GM is associated with T cell infiltration, expression of inducible nitric oxide synthase in microglia and profound accumulation of iron. Finally, it remains unclear whether and how deep GM involvement in MS contributes to the neurological disabilities including cognitive declines.

An increase in knowledge of MS pathology in WM and cortical and deep GM by new MRI techniques and more sensitive neuropathological technologies would result in elucidation of relevant pathogenic mechanisms associated with disease progression during the whole disease courses of MS.

\section{Competing interests None.}

Provenance and peer review Commissioned; internally peer reviewed.

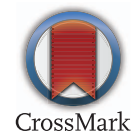

To cite Kawachi I. J Neurol Neurosurg Psychiatry 2014;85:1293.

Received 6 May 2014

Accepted 11 May 2014

Published Online First 4 June 2014

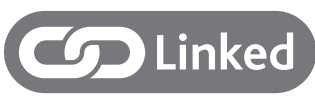

http://dx.doi.org/10.1136/jnnp-2014-307712

J Neurol Neurosurg Psychiatry 2014;85:1293. doi:10.1136/jnnp-2014-308221

\section{REFERENCES}

1 Geurts JJ, Barkhof F. Grey matter pathology in multiple sclerosis. Lancet Neurol 2008;7:841-51.

2 Kawachi I, Nishizawa M. Gray matter involvement in multiple sclerosis and neuromyelitis optica. Clin Exp Neuroimmunol 2014;5:69-76.

3 Calabrese M, Favaretto A, Martini V, et al. Grey matter lesions in MS: from histology to clinical implications. Prion 2013;7:20-7.

4 Minagar A, Barnett MH, Benedict RH, et al. The thalamus and multiple sclerosis: modern views on pathologic, imaging, and clinical aspects. Neurology 2013;80:210-9.

5 Lucchinetti CF, Popescu BF, Bunyan RF, et al. Inflammatory cortical demyelination in early multiple sclerosis. N Engl J Med 2011;365:2188-97.

6 Haider L, Simeonidou C, Steinberger G, et al. Multiple sclerosis deep grey matter: the relation between demyelination, neurodegeneration, inflammation and iron. J Neurol Neurosurg Psychiatry 2014;85:1375-84. 